\title{
Estudo da variação da viscosidade do vidro LKB a partir da adição de $\mathrm{Al}_{2} \mathrm{O}_{3}$
}

\author{
Study of the variation of the viscosity of LKB glass from the addition of $\mathrm{Al}_{2} \mathrm{O}_{3}$ \\ T. M. T. Nascimento ${ }^{1 *}$; J. A. L. S. Filho ${ }^{1}$; H. J. Khoury ${ }^{2}$; R. A. P. Oliveira ${ }^{1}$ \\ ${ }^{1}$ Colegiado da Pós-Graduação em Ciências dos Materiais, Universidade Federal do Vale do São Francisco, CEP \\ 48908-810, Juazeiro-BA, Brasil. \\ ${ }_{2}^{2}$ Departamento de Energia Nuclear, Universidade Federal de Pernambuco, CEP 50670-901, Recife-PE, Brasil.
}

*thais.muniz17@gmail.com

(Recebido em 07 de fevereiro de 2018; aceito em 13 de dezembro de 2018)

Ainda que o vidro seja um dos materiais mais antigos preparados pelo homem, ele apareceu apenas no século XX como um material que possui propriedades excelentes em áreas distintas. É um sólido amorfo com ausência de ordem a longo alcance e periodicidade, que possui transição vítrea. Pode ser obtido por várias formas, porém em grande parte é obtido pelo processo de fusão seguido do resfriamento rápido do fundido. Neste processo, a viscosidade determina as condições de fusão dos óxidos formadores do vidro, taxa de desvitrificação, etc. Este trabalho tem como objetivo avaliar a variação na viscosidade do vidro $\mathrm{LKB}\left(\mathrm{Li}_{2} \mathrm{CO}_{3}\right.$ $\left.-\mathrm{K}_{2} \mathrm{CO}_{3}-\mathrm{H}_{3} \mathrm{BO}_{3}\right)$ quando adicionado óxido de alumínio $\left(\mathrm{Al}_{2} \mathrm{O}_{3}\right)$ em diferentes proporções. A análise será efetuada utilizando o software SciGlass 7.0. Para tanto, quatro composições de vidros boratos foram desenvolvidas e estudadas. O cálculo da viscosidade em função da temperatura foi obtido para cada tipo de vidro a partir da composição dos óxidos em \% mol. Resultados preliminares mostraram que a adição do óxido de alumínio aumenta a viscosidade na matriz LKB. Além disso, foi possível preparar amostras vítreas com alta transparência e homogeneidade em todos os casos a partir da correlação entre a viscosidade e a temperatura.

Palavras-chave: vidro, viscosidade, óxido de alumínio.

Although glass is one of the oldest materials prepared by man, it appeared only in the twentieth century as a material that possesses excellent properties in distinct areas. It is an amorphous solid with absence of longrange order and periodicity, which has a glass transition. It can be obtained in various forms, but to a large extent it is obtained by the melting process followed by rapid cooling of the melt. In this process, the viscosity determines the melting conditions of the glass forming oxides, devitrification rate, etc. The objective of this work was to evaluate the variation of the viscosity of the $\mathrm{LKB}$ glass $\left(\mathrm{Li}_{2} \mathrm{CO}_{3}-\mathrm{K}_{2} \mathrm{CO}_{3}-\mathrm{H}_{3} \mathrm{BO}_{3}\right)$ when added aluminum oxide $\left(\mathrm{Al}_{2} \mathrm{O}_{3}\right)$ in different proportions. For this analysis the software SciGlass 7.0 was used. For this purpose, four compositions of borate glasses were developed and studied. The viscosity calculation as a function of temperature was obtained for each type of glass from the composition of the oxides in mol\%. Preliminary results showed that the addition of the aluminum oxide increases the viscosity in the LKB matrix. In addition, it was possible to prepare vitreous samples with high transparency and homogeneity in all cases from the correlation between viscosity and temperature.

Keywords: glass, viscosity, aluminum oxide.

\section{INTRODUÇÃO}

O vidro é um sólido, não cristalino, que apresenta transição vítrea. Em geral, os formadores vítreos fundem em temperaturas elevadas a $1.700{ }^{\circ} \mathrm{C}$, tornando o processo caro e complexo. Para reduzir custos e facilitar a produção, são acrescentados óxidos metálicos que reduzem o ponto de fusão. Desde a sua descoberta, vem se adequando em vários ambientes como vitrais de igrejas medievais, vidros planos de janelas, para-brisas de automóveis, lentes de óculos e fibra óptica utilizada para telecomunicações [1].

Embora o vidro seja um dos materiais mais antigos preparado pelo homem, somente a partir do século XX começou a ser largamente utilizado na área de tecnologia [2, 3], devido às suas propriedades ópticas não lineares, à capacidade de incorporar metais de transição, à resistência mecânica, sua estabilidade química, entre outros. 
Matrizes de vidros boratos dopadas ou co-dopadas com diferentes materiais, têm sido investigadas como dosímetros $[4,5,6]$. Entretanto, apresentam alta higroscopicidade, o que limita significativamente sua praticidade. Nesse sentido, a preparação de sistemas vítreos boratos contendo óxido de alumínio tem se destacado pela possibilidade de ser incorporado em grandes quantidades e estabilizar a estrutura do vidro. Além disso, pode aumentar a sua durabilidade química e diminuir a higroscopicidade [7].

Entre os diversos métodos de preparação dos materiais vítreos, aqui será destacado o processo tradicional de fusão e resfriamento rápido. Esse procedimento requer a seleção dos reagentes, cálculos estequiométricos, homogeneização, forno em alta temperatura e resfriamento rápido, suficiente para não ocorrer à cristalização do material. Segundo Hopper et al. (1974) [8], as condições mais favoráveis para a formação vítrea envolvem uma grande viscosidade no ponto da fase cristalina e/ou um rápido aumento de viscosidade quando a temperatura se aproxima do ponto de solidificação. Assim, pode-se dizer que a viscosidade do vidro é uma propriedade importante na otimização do processo de produção vítrea, pois influencia as condições de fusão dos óxidos formadores do vidro, temperatura máxima de utilização, taxa de desvitrificação, etc.

A partir das pesquisas desenvolvidas com materiais vítreos foi possível criar bases de dados que reúnem as diversas propriedades físicas e químicas. Os softwares Sciglass e o Interglade tem sido os mais utilizados para acessar bancos de dados destes materiais. O Sciglass utiliza modelos empíricos do banco de dados que possibilita a previsão de diferentes propriedades, tal como a correlação da viscosidade com a composição química do vidro. Já o Interglade utiliza a regressão linear para a previsão de propriedades dos materiais. Neste trabalho, optou-se pela utilização do Sciglass pela sua facilidade de utilização e possuir versão gratuita.

Chimanski et al. (2015) [9] utilizaram o software SciGlass para estudar a viscosidade de materiais vítreos dentários e encontrar um valor ideal de viscosidade para a temperatura de processamento de cada técnica usada na fabricação do material. A relação entre a composição e a viscosidade para fundir os óxidos de formação de vidros aluminosilicatos foi estudada por Leko e Mazurin (2003) [10] no SciGlass e percebeu-se que a viscosidade é predominantemente afetada pela quantidade de óxido de alumínio em relação aos óxidos alcalinos.

Yoshimura et. al. (2015) [11] investigaram a viscosidade do vidro composto por $45 \% \mathrm{SiO}_{2}, 25 \%$ $\mathrm{Al}_{2} \mathrm{O}_{3}, 15 \% \mathrm{La}_{2} \mathrm{O}_{3}$ e $15 \% \mathrm{TiO}_{2}(\mathrm{~mol} \%)$ por meio do software SciGlass. Para isso, adicionou diferentes proporções de óxidos alcalinos $\left(\mathrm{Li}_{2} \mathrm{O}, \mathrm{Na}_{2} \mathrm{O}, \mathrm{K}_{2} \mathrm{O}, \mathrm{Rb}_{2} \mathrm{O}\right.$ e $\left.\mathrm{Cs}_{2} \mathrm{O}\right)$ substituindo a sílica $\left(\mathrm{SiO}_{2}\right)$. As simulações mostraram que a viscosidade do material diminui com o aumento da temperatura e aumenta com o aumento da concentração dos óxidos alcalinos. Percebeu-se também uma dependência da viscosidade semelhante para todos os óxidos alcalinos até a adição de $25 \%$ molar.

Vale destacar que não há investigações na literatura que mostrem o $\mathrm{Al}_{2} \mathrm{O}_{3}$ como um componente principal da matriz vítrea LKB e por isso a importância desse trabalho é a possibilidade de desenvolver um material com baixa higroscopicidade e de grandes aplicações tecnológicas. Além disso, este trabalho propõe avaliar a viscosidade do vidro correlacionando com a temperatura de fusão suficiente para verter o fundido para todas as composições escolhidas. Alta temperatura de fusão e viscosidade do material inviabilizaria o processo de produção comercial e por isso pretendese sintetizar os vidros em temperaturas abaixo de $1000{ }^{\circ} \mathrm{C}$, visando facilidade e custo no processo.

\section{MATERIAL E MÉTODOS}

Quatro composições de vidros boratos foram desenvolvidas e estudadas diferenciando a porcentagem dos óxidos de alumínio, boro e lítio a fim de fazer uma análise comparativa da viscosidade.

Os reagentes utilizados na preparação das amostras, com suas respectivas purezas e procedências, estão descritos a seguir:

- Carbonato de lítio $\left(\mathrm{Li}_{2} \mathrm{CO}_{3}\right) 99 \%$ - MaxiFlux

- Carbonato de potássio $\left(\mathrm{K}_{2} \mathrm{CO}_{3}\right) 99,7 \%$ - Neon

- Ácido bórico $\left(\mathrm{H}_{3} \mathrm{BO}_{3}\right)$ 99,5\% - Nuclear

- Óxido de alumínio $\left(\mathrm{Al}_{2} \mathrm{O}_{3}\right) 98 \%$ - Sigma Aldrich 
As amostras foram nomeadas e serão tratadas ao longo do texto por: LKB, LKBAl-1, LKBAl-2 e LKBAl-3. A Tabela 1 mostra a composição dos óxidos em \% mol de cada vidro. Os cálculos estequiométricos foram feitos com o auxílio do software Mathcad 14.

Tabela 1 - Porcentagens em mol de cada vidro.

\begin{tabular}{c|cccc}
\hline Amostras & $\begin{array}{c}\mathrm{Li}_{2} \mathrm{O}_{3} \\
(\mathrm{~mol} \%)\end{array}$ & $\begin{array}{c}\mathrm{K}_{2} \mathrm{O}_{3} \\
(\mathrm{~mol} \%)\end{array}$ & $\begin{array}{c}\mathrm{B}_{2} \mathrm{O}_{3} \\
(\mathrm{~mol} \%)\end{array}$ & $\begin{array}{c}\mathrm{Al}_{2} \mathrm{O}_{3} \\
(\mathrm{~mol} \%)\end{array}$ \\
\hline LKB & 20,23 & 10,00 & 69,77 & - \\
\hline LKBAl-1 & 15,33 & 10,00 & 69,67 & 5,00 \\
\hline LKBAl-2 & 14,24 & 10,00 & 64,75 & 11,00 \\
\hline LKBAl-3 & 7,18 & 10,00 & 71,82 & 11,00 \\
\hline
\end{tabular}

Os estudos teóricos da viscosidade como função da temperatura foram calculados usando modelos empíricos que relacionam essa propriedade com a composição química do vidro, através da versão gratuita do software e banco de dados SciGlass 7.0 [9]. O cálculo dessa propriedade realizou-se por meio da calculadora do SciGlass. Valores de viscosidade $(\eta)$ dada em Pa.s foram calculados para cada tipo de vidro a partir da composição dos óxidos em $\%$ mol e um gráfico expresso na forma de $\log \eta$ em função da temperatura foi obtido.

A partir dos valores de viscosidade de cada uma das amostras, os reagentes de composição básica dos vidros, descritos anteriormente, foram misturados e homogeneizados em um almofariz com o auxílio de um pistilo, ambos de ágata, seguindo a proporção adequada a cada uma das amostras desejadas e levados à fusão em um cadinho de Pt:5\%Au para um forno resistivo por $1 \mathrm{~h}$ a $950{ }^{\circ} \mathrm{C}$, sem uso de atmosfera especial. $\mathrm{O}$ vidro foi obtido por resfriamento rápido do fundido, em molde de grafite na temperatura ambiente do laboratório.

Difrações de raios X (DRX) do pó foram realizadas a fim de confirmar a estrutura amorfa dos vidros. As análises foram realizadas no Centro de Tecnologias Estratégicas do Nordeste (CETENE), no Difratômetro de Raios X Bruker, modelo D8 Advance Davinci, tendo um tubo de $\mathrm{CuK}_{\alpha}(\lambda=1,5418 \AA$ ) como fonte de radiação, operando em $40 \mathrm{mV} / 40 \mathrm{~mA}$, com varredura contínua, à temperatura ambiente.

\section{RESULTADOS E DISCUSSÃO}

Os resultados de viscosidade em altas temperaturas para cada composição de vidro estudada estão apresentados na Tabela 2. Segundo Scholze (1991) [12], o ponto de fusão se dá a uma temperatura que corresponde à viscosidade de aproximadamente 1 Pa.s.

Para o vidro LKB, a temperatura de fusão em $825^{\circ} \mathrm{C}$ já é suficiente para produção do material. Porém, Marini et al. (2015) [13] produziram a matriz vítrea LKB com a mesma composição descrita neste trabalho durante $1 \mathrm{~h}$ de fusão a $950{ }^{\circ} \mathrm{C}$, temperatura esta que em comparação aos dados aqui apresentados, se encontra com viscosidade bem abaixo de 1 Pa.s, ou seja, de fácil manuseio para o resfriamento rápido do fundido. Não há relatos na literatura quanto a produção dos vidros LKBAl1, LKBAl-2 e LKBAl-3.

No presente trabalho, pretende-se otimizar o processo de produção e a meta principal é produzir todos os vidros numa temperatura abaixo de $1000^{\circ} \mathrm{C}$. Nesse sentido, é importante destacar que para a temperatura de $950{ }^{\circ} \mathrm{C}$, as amostras LKBAl-2 e LKBAl-3 possuem viscosidade correspondente a 5,62 Pa.s e 17,78 Pa.s, respectivamente, ambas acima de 1 Pa.s. 
Tabela 2- Viscosidade em altas temperaturas obtida a partir do SciGlass.

\begin{tabular}{cc|cc|cc|cc}
\hline \multicolumn{2}{c|}{ LKB } & & \multicolumn{2}{|c|}{ LKBAl-1 } & \multicolumn{2}{c|}{ LKBAl-2 } & \multicolumn{2}{c}{ LKBAl-3 } \\
\hline $\begin{array}{c}\text { Temperatura } \\
\left({ }^{\circ} \mathrm{C}\right)\end{array}$ & $\eta$ & Temperatura & $\eta$ & Temperatura & $\eta$ & Temperatura & $\eta$ \\
& $($ Pa.s $)$ & $\left({ }^{\circ} \mathrm{C}\right)$ & $($ Pa.s $)$ & $\left({ }^{\circ} \mathrm{C}\right)$ & $($ Pa.s $)$ & $\left({ }^{\circ} \mathrm{C}\right)$ & $($ Pa.s $)$ \\
\hline 750 & 4,90 & 750 & 40,73 & 750 & 954,99 & 750 & $3.090,30$ \\
775 & 2,40 & 775 & 18,62 & 800 & 177,83 & 800 & 616,60 \\
800 & 1,26 & 800 & 9,12 & 850 & 45,71 & 850 & 154,88 \\
825 & 0,72 & 825 & 4,90 & 900 & 14,79 & 900 & 48,98 \\
850 & 0,43 & 850 & 2,75 & 950 & 5,62 & 950 & 17,78 \\
875 & 0,27 & 875 & 1,62 & 1000 & 2,51 & 1000 & 7,41 \\
900 & 0,18 & 900 & 1,00 & 1050 & 1,26 & 1050 & 3,39 \\
925 & 0,12 & 925 & 0,63 & 1100 & 0,68 & 1100 & 1,70 \\
\hline 950 & 0,08 & 950 & 0,43 & 1150 & 0,39 & 1150 & 0,93 \\
975 & 0,06 & 975 & 0,29 & 1200 & 0,24 & 1200 & 0,52 \\
1000 & 0,04 & 1000 & 0,20 & 1250 & 0,15 & 1250 & 0,32 \\
1025 & 0,03 & 1025 & 0,15 & 1300 & 0,10 & 1300 & 0,20 \\
\hline
\end{tabular}

A Figura 1 mostra a curva da viscosidade expresso na forma de $\log \eta$ de todos os vidros estudados em função da temperatura. Se uma reta horizontal for traçada em $y=0$ pode-se observar que o vidro LKB apresenta a menor viscosidade, seguida da LKBAl-1, LKBAl-2 e LKBAl-3. Fica evidente que o aumento de óxido de alumínio na matriz LKB causa um aumento de viscosidade. Este fato corrobora com o relato de Lima et al. (2008) [14], no qual diz que o óxido de alumínio aumenta a viscosidade do vidro. Além disso, Leko e Mazurin (2003) [9] sugerem que as variações de viscosidade acontecem principalmente pela variação da quantidade de óxido de alumínio em relação aos óxidos alcalinos.

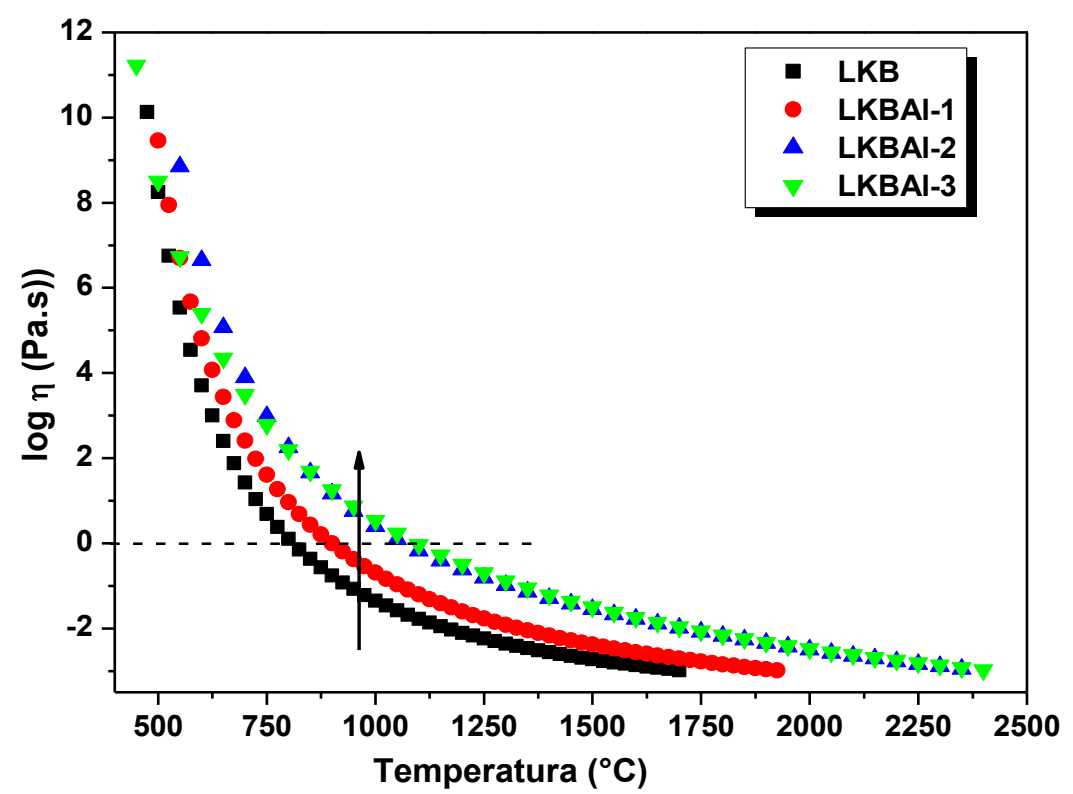

Figura 1 - Curva de viscosidade de todos os vidros estudados em função da temperatura. 
Com o intuito de fazer a correlação da viscosidade e temperatura de fusão adequada e ainda procurando atender ao objetivo de produzir as amostras abaixo de $1000{ }^{\circ} \mathrm{C}$, os reagentes de composição básica de todos os vidros, descritos anteriormente, foram levados à fusão em um cadinho de Pt:5\% Au num forno resistivo por $1 \mathrm{~h}$ a $950{ }^{\circ} \mathrm{C}$, sem uso de atmosfera especial. Todos os vidros foram obtidos por resfriamento rápido do fundido, em molde de grafite na temperatura ambiente do laboratório. Testes preliminares mostraram que teores maiores que $11 \%$ mol de $\mathrm{Al}_{2} \mathrm{O}_{3}$ produzem materiais cristalinos quando preparados a $950{ }^{\circ} \mathrm{C}$.

As amostras obtidas apresentaram alta transparência e homogeneidade em todos os casos, como mostra a Figura 2. Vale ressaltar que a amostra LKB (viscosidade de $0,08 \mathrm{~Pa} . \mathrm{s}$ a $950{ }^{\circ} \mathrm{C}$ ) apresentou maior facilidade durante a transferência para o molde de grafite se comparada com a amostra LKBAl-3 (viscosidade de 17,78 Pa.s a $950{ }^{\circ} \mathrm{C}$ ). Isso demonstra que quanto menor a viscosidade, mais fácil o processo de produção. Não há relatos na literatura quanto ao valor exato de viscosidade máxima para produção de vidro, os valores são sempre aproximados, como foi citado por Scholze (1991) [12]. Aqui, ficou comprovado que para a amostra LKBAl-2 e LKBAl-3, o valor de viscosidade 5,62 Pa.s e 17,78 Pa.s, respectivamente, são suficientes para fundir o material e produzir o vidro.

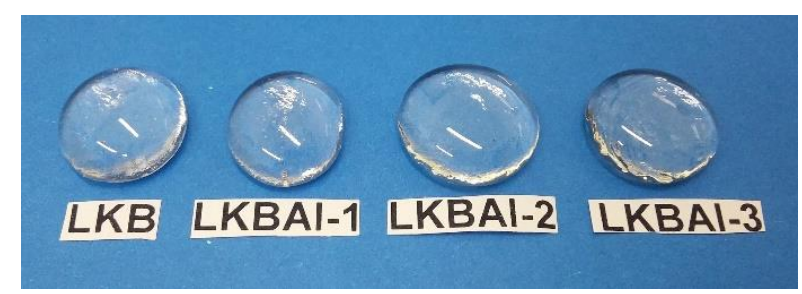

Figura 2 - Amostras vítreas produzidas.

A Figura 3 mostra os difratogramas obtidos para todas as amostras vítreas. Pode-se observar a formação de um halo em todas as amostras na faixa $2 \theta$, entre 20 e $35^{\circ}$, típico de materiais que possuem desordem a longos alcances. A ausência de picos de Bragg confirma a ausência de fases cristalinas.

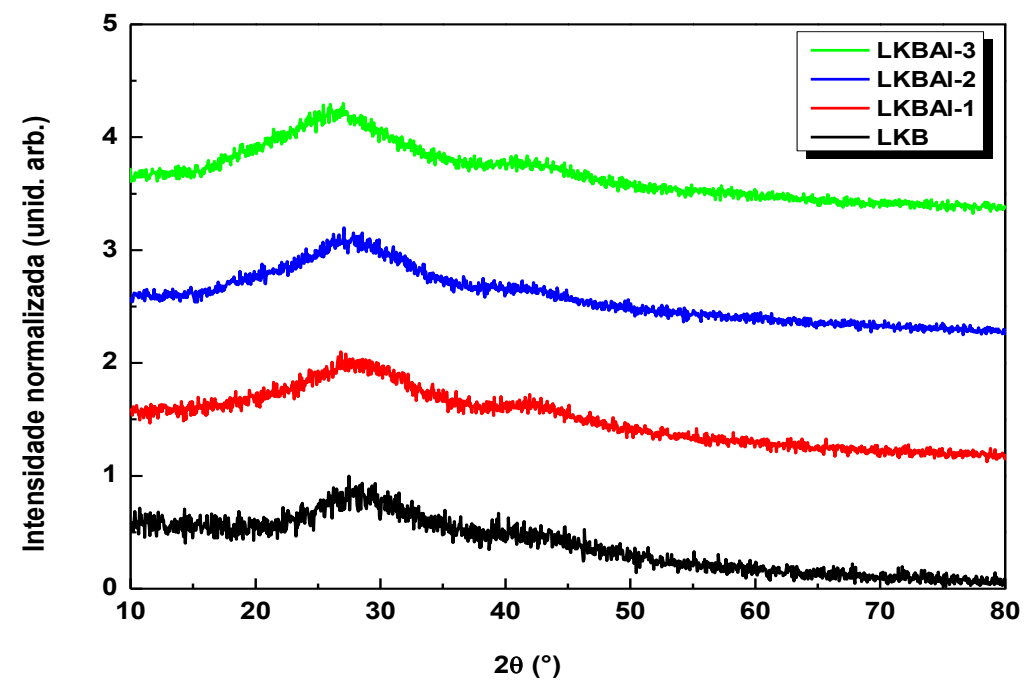

Figura 2 - Padrões de DRX de pó das amostras vítreas produzidas.

\section{CONCLUSÃO}

O estudo teórico obtido pelo software Sciglass demonstraram previsibilidade para determinar temperaturas características da viscosidade. Neste estudo, um novo sistema vítreo $\left(\mathrm{Li}_{2} \mathrm{O}_{3}-\mathrm{K}_{2} \mathrm{O}_{3}\right.$ $\mathrm{B}_{2} \mathrm{O}_{3}-\mathrm{Al}_{2} \mathrm{O}_{3}$ ) foi produzido pelo método de resfriamos rápido com sucesso a partir da correlação 
entre viscosidade e temperatura calculada pelo Sciglass. As amostras apresentaram alta transparência e homogeneidade, o que demonstra possibilidade de aplicação tecnológica.

Para o vidro LKB, a temperatura de fusão em $825^{\circ} \mathrm{C}$ já é suficiente para produção do material. As amostras LKBAl-1, LKBAL-2 e LKBAl-3 apresentaram um o valor ideal de temperatura de fusão à $950{ }^{\circ} \mathrm{C}$. Em todos os casos aqui estudados, é possível produzir as amostras abaixo de $1000{ }^{\circ} \mathrm{C}$, demonstrando facilidade e baixo custo no processo.

Todas as amostras que possuem óxido de alumínio da matriz LKB tiveram aumento de viscosidade. Sugere-se portanto, em trabalhos futuros, um estudo detalhado do novo sistema vítreo quanto a sua durabilidade química e higroscopicidade.

\section{AGRADECIMENTOS}

Os autores agradecem à Fundação de Amparo à Ciência e Tecnologia de Pernambuco (FACEPE) e à Fundação de Amparo à Pesquisa do Estado da Bahia (PABESB) pelo apoio financeiro. Ao Centro de Tecnologias Estratégicas do Nordeste (CETENE) pelo suporte na realização das análises de difração de raios X. Ao laboratório de mecânica da Universidade Federal do Vale do São Francisco (UNIVASF) pela disponibilidade na utilização dos fornos.

\section{REFERÊNCIAS BIBLIOGRÁFICAS}

1. Nascimento MLF. Brief history of the flat glass patent - Sixty years of the float process. World Patent Information. 2014 Sep;38:50-56, doi:10.1016/j.wpi.2014.04.006.

2. Lee SK, Eng PJ, Mao HK, Meng Y, Shu J. Structure of alkali borate glasses at high pressure: B and LiKedge inelastic $x$-ray scattering study. Phys Rev Lett. 2007 Mar;98:105502, doi:10.1103/PhysRevLett.98.105502.

3. Padmaja G, Kistaiah P. Optical characterization of $\mathrm{Mn}\left({ }^{2+}\right)$ : $\mathrm{Li}_{2} \mathrm{O}-\mathrm{K}_{2} \mathrm{O}-\mathrm{CdO}-\mathrm{B}_{2} \mathrm{O}_{3}$ glass system: Absorption edge, optical band gap, optical polarizability and optical basicity. IOP Conf. Series: Materials Science Engineer. 2009; 012040, doi:10.1088/1757-899X/2/1/012040.

4. Ayta WEF, Silva VA, Cano NF, Silva MAP, Dantas NO. Thermoluminescence, structural and magnetic properties of a $\mathrm{Li}_{2} \mathrm{O}-\mathrm{B}_{2} \mathrm{O}_{3}-\mathrm{Al}_{2} \mathrm{O}_{3}$ glass system doped with $\mathrm{LiF}$ and $\mathrm{TiO}_{2}$. J Lumines. 2011;131:1002-1006, doi:10.1016/j.jlumin.2011.01.011.

5. Ayta WEF, Silva VA, Dantas NO. Thermoluminescent properties of a $\mathrm{Li}_{2} \mathrm{O}-\mathrm{B}_{2} \mathrm{O}_{3}-\mathrm{Al}_{2} \mathrm{O}_{3}$ glass system doped with $\mathrm{CaF}_{2}$ and Mn. J Lumines. 2010 Jun;130(6):1032-1035, doi:10.1016/j.jlumin.2010.01.020

6. Hashim S, Alajerami YSM, Ghoshal SK, Saleh MA, Saripan MI, Kadir ABA, Bradley DA, Alzimami K. Dosimetric characteristics of LKB:Cu,P solid TL detector. Radiat Phys Chem. 2014;104:36-39, doi:10.1016/j.radphyschem.2013.12.015.

7. Ayta WEF, Silva VA, Cano NF, Silva MAP, Dantas NO. Thermoluminescence, structural and magnetic properties of a $\mathrm{Li}_{2} \mathrm{O}-\mathrm{B}_{2} \mathrm{O}_{3}-\mathrm{Al}_{2} \mathrm{O}_{3}$ glass system doped with $\mathrm{LiF}$ and $\mathrm{TiO}_{2}$. J Lumines. 2011;131:1002-1006, https://doi.org/10.1016/j.jlumin.2011.01.011.

8. Hopper RW, Scherer G, Uhlmann DR. Crystallization statistics, thermal history and glass formation. J Non-Crystalline Solids. 1974;15:45-62, doi:10.1016/0022-3093(74)90110-0.

9. Chimanski A, Cesar PF, Fredericci C, Yoshimura HN. Evaluation of glass viscosity of dental bioceramics by the SciGlass information system. Ceramics Int. 2015;41:10000-10009, doi:10.1016/j.ceramint.2015.04.082

10. Leko VK, Mazurin OV. Analysis of regularities in composition dependence of the viscosity for glassforming oxide melts: II. Viscosity of ternary alkali aluminosilicate melts. Glass Phys Chem. 2003;29:1627, doi:10.1023/A:1022301608310

11. Yoshimura HN, Chimanskia A, Cesarb PF. Systematic approach to preparing ceramic-glass composites with high translucency for dental restorations. Academy Dent Mater. 2015;31:1188-1197, doi:10.1016/j.dental.2015.06.015.

12. Scholze H. Glass: Nature, Structure and Properties. $1^{\text {st }}$ ed. New York: Springer-Verlag; 1991. 463 p.

13. Marini A, Valença JVB, Oliveira RAP, Souza SO, Cioli R, d'Errico F. Production and characterization of $\mathrm{H}_{3} \mathrm{BO}_{3}-\mathrm{Li}_{2} \mathrm{CO}_{3}-\mathrm{K}_{2} \mathrm{CO}_{3}-\mathrm{MgO}$ glass for dosimetry. Radiat Phys Chem. 2015;116:92-94, doi:10.1016/j.radphyschem.2015.04.011.

14. Lima ANC, Trommer RM, Zimmer A, Vicenzi J, Bragança SR, Boschetti J, Bergmann CP. Avaliação de revestimentos para proteção contra a descarbonetação de tijolos refratários $\mathrm{MgO}-\mathrm{C}$ durante o aquecimento de panelas de aciaria. R Matéria. 2008 Sep;13(3):488-494, doi:10.1590/S1517-70762008000300008. 